\title{
Three Positive Solutions of Multi-point BVPs for Difference Equations with the Nonlinearity Depending on $\Delta$-operator*
}

\author{
Yuji Liu
}

\begin{abstract}
This article deals with a class of discrete type boundary value problems. Sufficient conditions guaranteeing the existence of at least three positive solutions of this class of boundary value problems are established by using a fixed point theorem in cones in Banach spaces. An example is given to illustrate the main theorem.
\end{abstract}

\section{Introduction}

The existence of positive solutions of boundary value problems (BVPs for short) for difference equations were studied extensively, see the papers [1-4, $6-13,17,19,20,22,23]$ and the references therein. The approaches used are fixed point theorems in cones in Banach spaces (see [1-4, 6-13, 19-23]) and critical point theorems (see $[5,7]$ ).

There exist three kinds of processing methods to establish sufficient conditions for the existence of positive solutions of BVPs of difference equations by using the fixed point theorems in cones in Banach spaces.

Key Words: One-dimension $p$-Laplacian equation; multi-point boundary value problem; positive and negative coefficients.

2010 Mathematics Subject Classification: Primary 34B10; Secondary 34B15, 35B10.

Received: April, 2011.

Revised: April, 2011.

Accepted: February, 2012.

* Supported by Natural Science Foundation of Guangdong Province (No:S2011010001900) and the Guangdong Higher Education Foundation for High-level talents 
(i) For BVPs of difference equations without $p$-Laplacian, see [1-3, 6, 8, $10,19,20,22,23]$, by using the explicit expressions of the associated Green's functions, one transforms the BVPs into integral equations and operators in Banach spaces are defined, then the fixed point theorems are used to get the positive solutions;

(ii) For BVPs of difference equations with $p$-Laplacian, see $[5,9,12$, $13,17,18]$, by using some of the boundary condition such as $\Delta x(0)=0$, the BVPs are transformed into integral equations and operators in Banach spaces are defined. Then the fixed point theorems are used to get the positive solutions;

(iii) For multi-point BVPs of difference equations with $p$-Laplacian, see [14]. The difficult to study this kind of BVPs comes from that it is not easy to establish the associated Green's functions or transform BVPs into integral equations.

In recent paper [14], the author overcomed the difficulty mentioned above and studied the following multi-point BVP for the second order $p$-Laplacian difference equation with its nonlinearity $f$ depending on $\Delta x(n)$, i.e. the BVP of the form

$$
\left\{\begin{array}{l}
\Delta[\phi(\Delta x(n))]+f(n, x(n), \Delta x(n))=0, n \in[0, N], \\
x(0)=\sum_{i=1}^{m} \alpha_{i} x\left(n_{i}\right), \\
x(N+2)=\sum_{i=1}^{m} \beta_{i} x\left(n_{i}\right),
\end{array}\right.
$$

where $N>1$ an integer, $0<n_{1}<\cdots<n_{m}<N+2, \alpha_{i}, \beta_{i} \geq 0$ for all $i=1, \cdots, m, f$ is continuous and nonnegative, $\phi$ is called $p$-Laplacian, $\phi(x)=|x|^{p-2} x$ for $x \neq 0$ and $\phi(0)=0$ with $p>1$. Sufficient conditions for the existence of at least three positive solutions of $\mathrm{BVP}(1)$ were established. One finds that the coefficients $\alpha_{i}, \beta_{i}(i=1,2, \cdots, m)$ in BCs are all nonnegative.

Ma in $[15,16]$ studied the following BVP

$$
\left\{\begin{array}{l}
{\left[p(t) x^{\prime}(t)\right]^{\prime}-q(t) x(t)+f(t, x(t))=0, \quad t \in(0,1)} \\
\alpha x(0)-\beta p(0) x^{\prime}(0)=\sum_{i=1}^{m} a_{i} x\left(\xi_{i}\right), \\
\gamma x(1)+\delta p(1) x^{\prime}(1)=\sum_{i=1}^{m} b_{i} x\left(\xi_{i}\right)
\end{array}\right.
$$

where $0<\xi_{1}<\cdots<\xi_{m}<1, \alpha, \beta, \gamma, \delta \geq 0, a_{i}, b_{i} \geq 0$ with $\rho=\gamma \beta+\alpha \gamma+$ $\alpha \delta>0$. By using Green's functions (an abstract not an explicit ones, which complicate the studies of $\mathrm{BVP}(2))$ and Guo-Krasnoselskii fixed point theorem, the existence and multiplicity of positive solutions for $\mathrm{BVP}(2)$ were given. 
It is easy to see that the discrete analogue of BVP (2) is as follows:

$$
\left\{\begin{array}{l}
\Delta(p(n) \phi(\Delta x(n)))-q(n) x(n)+f(n, x(n))=0, n \in[0, T], \\
\alpha x(0)-\beta \Delta x(0)=\sum_{i=1}^{m} a_{i} x\left(n_{i}\right), \\
\gamma x(T+2)+\delta \Delta x(T+1)=\sum_{i=1}^{m} b_{i} x\left(n_{i}\right) .
\end{array}\right.
$$

The coefficients of the BCs of the right side are nonnegative, i.e. $a_{i} \geq 0$ and $b_{i} \geq 0$ for all $i \in[1, m]$.

In $\mathrm{Xu}^{\prime}$ s paper [21], by using Guo-Krasnoselskii fixed point theorem, the existence results of at least one or two positive solutions of the following problem

$$
\left\{\begin{array}{l}
{\left[\phi\left(x^{\prime}(t)\right)\right]^{\prime}+a(t) f(x(t))=0, \quad t \in(0,1),} \\
x^{\prime}(0)=\sum_{i=1}^{m} a_{i} x^{\prime}\left(\xi_{i}\right), \\
x(1)=\sum_{i=1}^{k} b_{i} x\left(\xi_{i}\right)-\sum_{i=k+1}^{s} b_{i} x\left(\xi_{i}\right)-\sum_{i=s+1}^{m-2} b_{i} x^{\prime}\left(\xi_{i}\right)
\end{array}\right.
$$

was obtained, where all $a_{i} \geq 0$ and $b_{i} \geq 0,0<\xi_{1}<\xi_{2}<\cdots<x_{m-2}<1, a(t)$ and $f(x)$ are nonnegative and continuous functions. The discrete analogue of $\operatorname{BVP}(4)$ is as follows

$$
\left\{\begin{array}{l}
{[\phi(\Delta x(n))]^{\prime}+a(n) f(x(n))=0, \quad n \in[0, N],} \\
\Delta x(0)=\sum_{i=1}^{m} a_{i} \Delta x\left(n_{i}\right), \\
x(N+2)=\sum_{i=1}^{k} b_{i} x\left(n_{i}\right)-\sum_{i=k+1}^{s} b_{i} x\left(n_{i}\right)-\sum_{i=s+1}^{m-2} b_{i} \Delta x^{\prime}\left(n_{i}\right) .
\end{array}\right.
$$

Besides [14], there exist no other papers discussed the existence of multiple positive solutions of multi-point BVPs for $p$-Laplacian difference equations even $\operatorname{BVP}(3)$ and $\operatorname{BVP}(5)$ in which the nonlinearity $f$ is independent on $\Delta x(n)$.

Motivated by the reason and papers mentioned above, the purpose of this paper is to investigate the multi-point BVP for the second order $p$-Laplacian difference equation whose nonlinear term is dependent on $\Delta x(n)$, i.e. the BVP of the form

$$
\left\{\begin{array}{l}
\Delta[p(n) \phi(\Delta x(n))]+f(n, x(n), \Delta x(n))=0, \quad n \in[0, N], \\
\alpha x(0)-\beta \Delta x(0)=-a x\left(n_{1}\right)+b x\left(n_{2}\right)+a_{1} \Delta x\left(n_{1}\right)-b_{1} \Delta x\left(n_{2}\right), \\
\Delta x(N+1)=c \Delta x\left(m_{1}\right)-d \Delta x\left(m_{2}\right),
\end{array}\right.
$$

where $N>1$ an integer, $0<n_{1}<n_{2}<N+2$ and $0<m_{1}<m_{2}<N+2$, $\alpha, \beta, \gamma, \delta, a, b, c, d, a_{1}, b_{1} \geq 0, f$ is continuous and nonnegative, $\phi$ is called $p$-Laplacian, $\phi(x)=|x|^{p-2} x$ with $p>1$, its inverse function is denoted by $\phi^{-1}(x)=|x|^{q-2} x$ with $1 / p+1 / q=1$.

Sufficient conditions for the existence of at least three positive solutions of $\operatorname{BVP}(6)$ are established. Note that the coefficients of the right sides in boundary conditions in $\operatorname{BVP}(6)$ have negative and positive signs. The methods and 
the results in this paper, which are not based upon the Green's functions, are different from those in known papers. The results improve and generalize some known theorems.

The methods can be extended to establish existence results for positive solutions of the more general BVP of the form

$$
\left\{\begin{array}{l}
\Delta[p(n) \phi(\Delta x(n))]+f(n, x(n), \Delta x(n))=0, \quad n \in[0, N], \\
\alpha x(0)-\beta \Delta x(0)=-\sum_{i=1}^{m} a_{i} x\left(n_{i}\right) \\
\quad+\sum_{i=m+1}^{n} a_{i} x\left(n_{i}\right)+\sum_{i=1}^{m} b_{i} \Delta x\left(n_{i}\right)-\sum_{i=m+1}^{n} b_{i} \Delta x\left(n_{i}\right), \\
\Delta x(N+1)=\sum_{i=1}^{k} c_{i} \Delta x\left(m_{i}\right)-\sum_{i=k+1}^{n} c_{i} \Delta x\left(m_{i}\right) .
\end{array}\right.
$$

The readers should try to do it further.

The technique used in this paper is very valuable and demonstrates a method of dealing with existence of solutions arguments for measure chain arguments for p-Laplacian.

The remainder of this paper is organized as follows: the main results and examples to illustrate it are presented in Section 2, the lemmas and proofs of the main theorems are given in Section 3.

\section{Main Results and Examples}

In this section, we present the main result and give an example to illustrate the efficiency of the main theorem. Suppose that

(A1) $\alpha>0, \beta \geq 0, a \geq 0, b \geq 0, a_{1} \geq 0, b_{1} \geq 0$ with $0 \leq b-a<\alpha$, and

$$
0 \leq b_{1} \phi^{-1}\left(\frac{p(0)}{p\left(n_{2}\right)}\right)-a_{1} \phi^{-1}\left(\frac{p(0)}{p\left(n_{1}\right)}\right)<\beta
$$

and

$$
0 \leq c \phi^{-1}\left(\frac{p(N+1)}{p\left(m_{1}\right)}\right)-d \phi^{-1}\left(\frac{p(N+1)}{p\left(m_{2}\right)}\right)<1
$$

(A2) $p:[0, N+1] \rightarrow(0,+\infty)$ and there exists a $k \in[1, N+1]$ such that $p(s) \geq p(j)$ for all $s \in[k, N+1]$ and $j \in[0, k-1]$;

(A3) $f:[0, N] \times[0,+\infty) \times R \rightarrow[0,+\infty)$ is a continuous function. 
For a group of positive constants $e_{1}, e_{2}, c$, denote

$$
\begin{aligned}
\delta & =\phi\left(\frac{1+d \phi^{-1}\left(\frac{p(N+1)}{p\left(m_{2}\right)}\right)}{c \phi^{-1}\left(\frac{p(N+1)}{p\left(m_{1}\right)}\right)}\right)-1 \\
Q & =\phi\left(\frac{\alpha c}{\alpha N+2 \alpha+\beta+a n_{1}+b n_{2}+a_{1}+b_{1}}\right) \frac{\delta \min _{n \in[0, N]} p(n)}{(1+\delta)(N+1)} \\
W & =\phi\left(\frac{(N+2) e_{2}}{k \sum_{i=0}^{k} \phi^{-1}\left(\frac{N-k+1}{p(i)}\right)}\right) ; \\
E & =\phi\left(\frac{\alpha e_{1}}{\alpha N+2 \alpha+\beta+a n_{1}+b n_{2}+a_{1}+b_{1}}\right) \frac{\delta \min _{n \in[0, N]} p(n)}{(1+\delta)(N+1)}
\end{aligned}
$$

Theorem 2.1. Suppose that $(A 1)-(A 3)$ hold. Furthermore, suppose there exist positive constants $e_{1}, e_{2}, c_{1}$ such that $Q>W$ and

$$
0<e_{1}<e_{2}<\frac{N+2}{k} e_{2}<c_{1}
$$

and

(A4) $f(t, u, v)<Q$ for all $t \in[0, N], u \in\left[0, c_{1}\right], v \in\left[-c_{1}, c_{1}\right]$;

(A5) $f(t, u, v) \geq W$ for all $t \in[k, N], u \in\left[e_{2}, \frac{N+2}{k} e_{2}\right], v \in\left[-c_{1}, c_{1}\right]$;

(A6) $f(t, u, v) \leq E$ for all $t \in[0, N], u \in\left[0, e_{1}\right], v \in\left[-c_{1}, c_{1}\right]$.

Then $\operatorname{BVP}(6)$ has three increasing positive solutions $x_{1}, x_{2}$ and $x_{3}$ such that

$$
x_{1}(N+2)<e_{1}, x_{2}(k)>e_{2}, x_{3}(N+2)>e_{1}, x_{3}(k)<e_{2} .
$$

Now, we present an example, which can not be covered by known theorems, to illustrate Theorem 2.1.

Example 2.1. Consider the following multi-point BVP

$$
\left\{\begin{array}{l}
\left.\Delta[p(n+1)(\Delta x(n)))^{3}\right]+f(n, x(n), \Delta x(n))=0, \quad n \in[0,99] \\
x(0)-\Delta x(0)=\frac{1}{2} x(9)-\frac{1}{3} x(19)-\frac{1}{8} \Delta x(9)+\frac{1}{2} \Delta x(19), \\
\Delta x(101)=-\frac{1}{4} \Delta x(9)+\frac{1}{4} \Delta x(19)
\end{array}\right.
$$

where $f:[0,99] \times[0,+\infty) \times R \rightarrow[0,+\infty)$ is defined by

$$
f(n, x, y)=\frac{n}{10^{19}}+f_{0}(x)+\frac{|y|}{2 \times 10^{39}}, n \in[0,99], x \geq 0, y \in R
$$


where

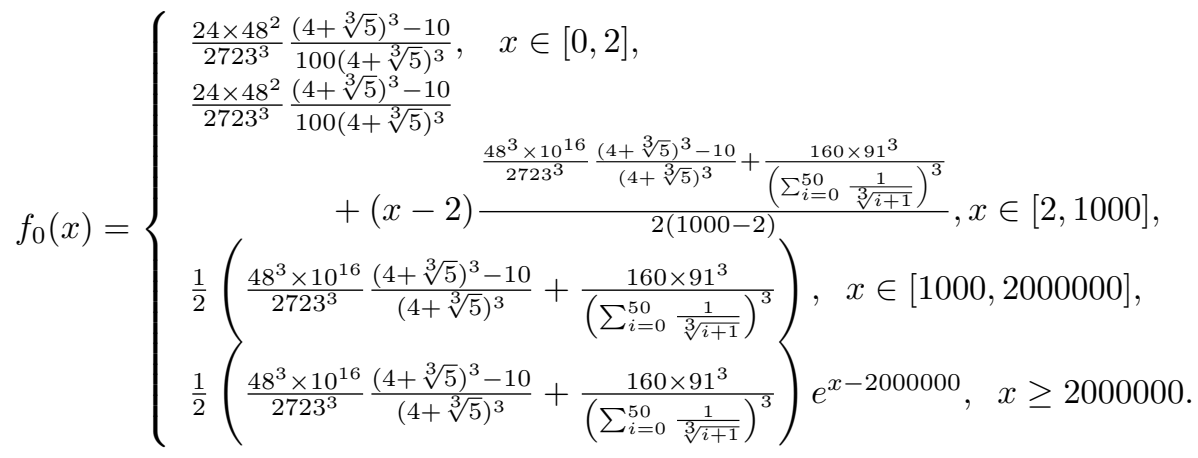

It is easy to see that $f$ is a continuous function.

Corresponding to $\operatorname{BVP}(6)$, one sees that $\phi(x)=x^{3}, p(n)=n+1, N=99$, $\alpha=1, \beta=1 n_{1}=9, n_{2}=19, m_{1}=9, m_{2}=19, a=1 / 2, b=1 / 3, a_{1}=$ $1 / 8, b_{1}=1 / 2, c=1 / 4, d=1 / 4$.

Use Theorem 2.1. Choose constants $k=50$. One sees that $(A 1)-(A 3)$ hold.

Choose $e_{1}=2, e_{2}=1000, c_{1}=2000000$, then

$$
\begin{aligned}
\delta & =\phi\left(\frac{1+d \phi^{-1}\left(\frac{p(N+1)}{p\left(m_{2}\right)}\right)}{c \phi^{-1}\left(\frac{p(N+1)}{p\left(m_{1}\right)}\right)}\right)-1=\frac{(4+\sqrt[3]{5})^{3}-10}{10}, \\
Q & =\phi\left(\frac{\alpha c_{1}}{\alpha N+2 \alpha+\beta+a n_{1}+b n_{2}+a_{1}+b_{1}}\right) \frac{\delta \min _{n \in[0, N]} p(n)}{(1+\delta)(N+1)} \\
& =\frac{48^{3} \times 10^{16}}{2723^{3}} \frac{(4+\sqrt[3]{5})^{3}-10}{(4+\sqrt[3]{5})^{3}} ; \\
W & =\phi\left(\frac{(N+2) e_{2}}{k \sum_{i=0}^{k} \phi^{-1}\left(\frac{N-k+1}{p(i)}\right)}\right)=\frac{160 \times 91^{3}}{\left(\sum_{i=0}^{50} \frac{1}{\sqrt[3]{i+1}}\right)^{3}} ; \\
E & =\phi\left(\frac{\alpha e_{1}}{\alpha N+2 \alpha+\beta+a n_{1}+b n_{2}+a_{1}+b_{1}}\right) \frac{\delta \min _{n \in[0, N]} p(n)}{(1+\delta)(N+1)} \\
& =\frac{48^{3}}{2723^{3}} \frac{(4+\sqrt[3]{5})^{3}-10}{100(4+\sqrt[3]{5})^{3}} .
\end{aligned}
$$

It is easy to see that $Q>W$ and

$$
c_{1}>\frac{N+2}{k} e_{2}>e_{2}>e_{1}>0 .
$$


One sees that

$$
\begin{aligned}
& f_{0}(u) \leq \frac{48^{3} \times 10^{16}}{2723^{3}} \frac{(4+\sqrt[3]{5})^{3}-10}{(4+\sqrt[3]{5})^{3}} \text { for all } u \in[0,2000000] \\
& f_{0}(u) \geq \frac{160 \times 91^{3}}{\left(\sum_{i=0}^{50} \frac{1}{\sqrt[3]{i+1}}\right)^{3}} \text { for all } u \in[1000,2020] \\
& f_{0}(u) \leq \frac{48^{3}}{2723^{3}} \frac{(4+\sqrt[3]{5})^{3}-10}{100(4+\sqrt[3]{5})^{3}} \text { for all } u \in[0,2]
\end{aligned}
$$

It follows that

$f(t, u, v) \leq \frac{48^{3} \times 10^{16}}{2723^{3}} \frac{(4+\sqrt[3]{5})^{3}-10}{(4+\sqrt[3]{5})^{3}}$ for all $t \in[0,99], u \in[0,2000000], v \in$ $[-2000000,2000000]$;

$$
f(t, u, v) \geq \frac{160 \times 91^{3}}{\left(\sum_{i=0}^{50} \frac{1}{\sqrt[3]{i+1}}\right)^{3}} \text { for all } t \in[50,99], u \in[1000,2020], v \in[-2000000,
$$
2000000]

$f(t, u, v) \leq \frac{48^{3}}{2723^{3}} \frac{(4+\sqrt[3]{5})^{3}-10}{100(4+\sqrt[3]{5})^{3}}$ for all $t \in[0,99], u \in[0,2]$ and $v \in[-2000000$, 2000000];

Hence $(A 4),(A 5),(A 6)$ hold. Then applying Theorem 2.1 BVP $(8)$ has at least three solutions $x_{1}, x_{2}, x_{3}$ such that $x_{1}(101)<6, x_{2}(50)>1004, x_{3}(101)>$ $6, x_{3}(50)<1004$.

Remark 1. One can not get three solutions of BVP (8) by applying the theorems obtained in papers $[2,6-8,10,12-14,17,19-22]$.

\section{Proofs of Theorem 2.1}

In this section, we first present some background definitions in Banach spaces, state the important three fixed point theorem and lemmas. Then the main results are proved.

Definition 3.1. Let $X$ be a semi-ordered real Banach space. The nonempty convex closed subset $P$ of $X$ is called a cone in $X$ if $a x \in P$ and $x+y \in P$ for all $x, y \in P$ and $a \geq 0, x \in X$ and $-x \in X$ imply $x=0$.

Definition 3.2. A map $\psi: P \rightarrow[0,+\infty)$ is a nonnegative continuous concave or convex functional map provided $\psi$ is nonnegative, continuous and satisfies $\psi(t x+(1-t) y) \geq t \psi(x)+(1-t) \psi(y)$, or $\psi(t x+(1-t) y) \leq t \psi(x)+$ $(1-t) \psi(y)$, for all $x, y \in P$ and $t \in[0,1]$.

Definition 3.3. An operator $T ; X \rightarrow X$ is completely continuous if it is continuous and maps bounded sets into pre-compact sets.

Let $c_{1}, c_{2}, c_{3}, c_{4}, c_{5}>0$ be positive constants, $\alpha_{1}, \alpha_{2}$ be two nonnegative continuous concave functionals on the cone $P, \beta_{1}, \beta_{2}, \beta_{3}$ be three nonnegative 
continuous convex functionals on the cone $P$. Define the convex sets as follows:

$$
\begin{aligned}
& P_{c_{5}}=\left\{x \in P:\|x\|<c_{5}\right\}, \\
& P\left(\beta_{1}, \alpha_{1} ; c_{2}, c_{5}\right)=\left\{x \in P: \alpha_{1}(x) \geq c_{2}, \beta_{1}(x) \leq c_{5}\right\}, \\
& P\left(\beta_{1}, \beta_{3}, \alpha_{1} ; c_{2}, c_{4}, c_{5}\right)=\left\{x \in P: \alpha_{1}(x) \geq c_{2}, \beta_{3}(x) \leq c_{4}, \beta_{1}(x) \leq c_{5}\right\}, \\
& Q\left(\beta_{1}, \beta_{2} ;, c_{1}, c_{5}\right)=\left\{x \in P: \beta_{2}(x) \leq c_{1}, \beta_{1}(x) \leq c_{5}\right\}, \\
& Q\left(\beta_{1}, \beta_{2}, \alpha_{2} ; c_{3}, c_{1}, c_{5}\right)=\left\{x \in P: \alpha_{2}(x) \geq c_{3}, \beta_{2}(x) \leq c_{1}, \beta_{1}(x) \leq c_{5}\right\} .
\end{aligned}
$$

Lemma 3.1[3, 11]. Let $X$ be a real Banach space, $P$ be a cone in $X$. $\alpha_{1}, \alpha_{2}$ be two nonnegative continuous concave functionals on the cone $P$, $\beta_{1}, \beta_{2}, \beta_{3}$ be three nonnegative continuous convex functionals on the cone $P$. Then $T$ has at least three fixed points $y_{1}, y_{2}$ and $y_{3}$ such that

$$
\beta_{2}\left(y_{1}\right)<c_{1}, \alpha_{1}\left(y_{2}\right)>c_{2}, \beta_{2}\left(y_{3}\right)>c_{1}, \alpha_{1}\left(y_{3}\right)<c_{2}
$$

if

(1) $T: X \rightarrow X$ is a completely continuous operator;

(2) there exist constant $M>0$ such that $\alpha_{1}(x) \leq \beta_{2}(x),\|x\| \leq M \beta_{1}(x)$ for all $x \in P$;

(3) there exist positive numbers $c_{1}, c_{2}, c_{3}, c_{4}, c_{5}$ with $c_{1}<c_{2}$ such that

(C1) $T \overline{P_{c_{5}}} \subset \overline{P_{c_{5}}}$;

(C2) $\left\{y \in P\left(\beta_{1}, \beta_{3}, \alpha_{1} ; c_{2}, c_{4}, c_{5}\right) \mid \alpha_{1}(x)>c_{2}\right\} \neq \emptyset$ and

$\alpha_{1}(T x)>c_{2}$ for every $x \in P\left(\beta_{1}, \beta_{3}, \alpha_{1} ; c_{2}, c_{4}, c_{5}\right)$;

(C3) $\left\{y \in Q\left(\beta_{1}, \beta_{2}, \alpha_{2} ; c_{3}, c_{1}, c_{5}\right) \mid \beta_{2}(x)<c_{1}\right\} \neq \emptyset$ and

$\beta_{2}(T x)<c_{1}$ for every $x \in Q\left(\beta_{1}, \beta_{2}, \alpha_{2} ; c_{3}, c_{1}, c_{5}\right)$;

(C4) $\alpha_{1}(T y)>c_{2}$ for $y \in P\left(\beta_{1}, \alpha_{1} ; c_{2}, c_{5}\right)$ with $\beta_{3}(T y)>c_{4}$; (C5) $\beta_{2}(T x)<c_{1}$ for each $x \in Q\left(\beta_{1}, \beta_{2} ; c_{1}, c_{5}\right)$ with $\alpha_{2}(T x)<c_{3}$.

Choose $X=R^{N+3}$. We call $x \leq y$ for $x, y \in X$ if $x(n) \leq y(n)$ for all $n \in[0, N+2]$, define the norm

$$
\|x\|=\max \left\{\max _{n \in[0, N+2]}|x(n)|, \max _{n \in[0, N+1]}|\Delta x(n)|\right\} .
$$

It is easy to see that $X$ is a semi-ordered real Banach space.

Choose

$$
P=\left\{\begin{array}{c}
x(n) \text { is increasing and positive on }[0, N+2], \\
x(k) \geq \frac{k}{N+2}|x(N+2)|, k \text { is defined in }(A 2), \\
x \in X: \quad \alpha x(0)-\beta \Delta x(0)=-a x\left(n_{1}\right)+b x\left(n_{1}\right) \\
+a_{1} \Delta x\left(n_{1}\right)-b_{1} \Delta x\left(n_{2}\right), \\
\Delta x(N+1)=c \Delta x\left(m_{1}\right)-d \Delta x\left(m_{2}\right) .
\end{array}\right\}
$$


It is easy to see that $P$ is a cone in $X$.

Define the functionals on $P$ by

$$
\begin{aligned}
\beta_{1}(y) & =\max _{n \in[0, N+1]}|\Delta y(n)|, y \in P, \\
\beta_{2}(y) & =\max _{n \in[0, N+2]}|y(n)|, y \in P, \\
\beta_{3}(y) & =\max _{n \in[k, N+2]}|y(n)|, y \in P, \\
\alpha_{1}(y) & =\min _{t \in[k, N+2]}|y(n)|, y \in P, \\
\alpha_{2}(y) & =\min _{t \in[k, N+2]}|y(n)|, y \in P .
\end{aligned}
$$

Lemma 3.2. Suppose that $(A 1)-(A 3)$ hold. If $x \in X$ is a solution of $\operatorname{BVP}(6)$, then

(i) $p(n) \phi(\Delta x(n))$ is decreasing on $[0, N+1]$;

(ii) $\Delta x(n) \geq 0$ for all $n \in[0, N+1]$;

(iii) $x(n)>0$ for all $n \in[1, N+1]$;

(iv) $x(k) \geq \frac{k}{N+2} x(N+2) \mid$;

(v) it holds that

$$
x(N+2) \leq\left(N+2+\frac{\beta+a n_{1}+b n_{2}+a_{1}+b_{1}}{\alpha}\right) \max _{s \in[0, N+1]}|\Delta x(s)| .
$$

Proof. The proofs are omitted.

Lemma 3.3. Let $\delta$ be defined in Section 2 and denote

$$
\sigma_{f}=\phi^{-1}\left(\frac{\sum_{j=0}^{N} f(j, x(j), \Delta x(j))}{\delta p(N+1)}\right) .
$$

Suppose that $(A 1),(A 2)$ hold. If $x \in X$ if a solution of $\operatorname{BVP}(6)$, then

$$
x(n)=B_{x}+\sum_{i=0}^{n-1} \phi^{-1}\left(\frac{p(N+1) \phi\left(A_{x}\right)+\sum_{j=n}^{N} f(j, x(j), \Delta x(j))}{p(n)}\right),
$$

where $A_{x}$ satisfies

$$
\begin{aligned}
A_{x}= & c \phi^{-1}\left(\frac{p(N+1) \phi\left(A_{x}\right)+\sum_{j=m_{1}}^{N} f(j, x(j), \Delta x(j))}{p\left(m_{1}\right)}\right) \\
& -d \phi^{-1}\left(\frac{p(N+1) \phi\left(A_{x}\right)+\sum_{j=m_{2}}^{N} f(j, x(j), \Delta x(j))}{p\left(m_{2}\right)}\right) \in\left[0, \sigma_{f}\right],
\end{aligned}
$$


and $B_{x}$ satisfy the equalities:

$$
\begin{aligned}
B_{x}= & \frac{1}{\alpha+a-b}\left[\beta \phi^{-1}\left(\frac{p(N+1) \phi\left(A_{x}\right)+\sum_{j=0}^{N} f(j, x(j), \Delta x(j))}{p(0)}\right)\right. \\
& -a \sum_{i=0}^{n_{1}-1} \phi^{-1}\left(\frac{p(N+1) \phi\left(A_{x}\right)+\sum_{j=i}^{N} f(j, x(j), \Delta x(j))}{p(i)}\right) \\
& +b \sum_{i=0}^{n_{2}-1} \phi^{-1}\left(\frac{p(N+1) \phi\left(A_{x}\right)+\sum_{j=i}^{N} f(j, x(j), \Delta x(j))}{p(i)}\right) \\
& +a_{1} \phi^{-1}\left(\frac{p(N+1) \phi\left(A_{x}\right)+\sum_{j=n_{1}}^{N} f(j, x(j), \Delta x(j))}{p\left(n_{1}\right)}\right) \\
& \left.-b_{1} \phi^{-1}\left(\frac{p(N+1) \phi\left(A_{x}\right)+\sum_{j=n_{2}}^{N} f(j, x(j), \Delta x(j))}{p\left(n_{2}\right)}\right)\right] .
\end{aligned}
$$

Proof. Since $x \in X$ is a solution of $\operatorname{BVP}(6)$, we get that there exist constants $A_{x}$ and $B_{x}$ such that

$$
\Delta x(n)=\phi^{-1}\left(\frac{p(N+1) \phi\left(A_{x}\right)+\sum_{j=n}^{N+1} f(j, x(j), \Delta x(j))}{p(n)}\right),
$$

and

$$
x(n)=B_{x}+\sum_{i=0}^{n-1} \phi^{-1}\left(\frac{p(N+1) \phi\left(A_{x}\right)+\sum_{j=i}^{N+1} f(j, x(j), \Delta x(j))}{p(i)}\right) .
$$

It follows from the BCs in (6) that $A_{x}$ and $B_{x}$ are defined. Now we prove that

$$
A_{x} \in\left[0, \sigma_{f}\right] .
$$

It follows from Lemma 3.3 that $A_{x} \geq 0$. Suppose that $A_{x} \neq 0$. Let

$$
\begin{aligned}
F(s)= & 1-c \phi^{-1}\left(\frac{p(N+1)+\frac{1}{\phi(s)} \sum_{j=m_{1}}^{N} f(j, x(j), \Delta x(j))}{p\left(m_{1}\right)}\right) \\
& +d \phi^{-1}\left(\frac{p(N+1)+\frac{1}{\phi(s)} \sum_{j=m_{2}}^{N} f(j, x(j), \Delta x(j))}{p\left(m_{2}\right)}\right) .
\end{aligned}
$$


If $s>0$, it is easy to see that

$$
\begin{aligned}
F(s)> & 1-c \phi^{-1}\left(\frac{p(N+1)+\frac{1}{\phi(s)} \sum_{j=m_{1}}^{N} f(j, x(j), \Delta x(j))}{p\left(m_{1}\right)}\right) \\
& +d \phi^{-1}\left(\frac{p(N+1)}{p\left(m_{2}\right)}\right)=: G(s) .
\end{aligned}
$$

One finds that

$$
\lim _{s \rightarrow+\infty} G(s)=1-c \phi^{-1}\left(\frac{p(N+1)}{p\left(m_{1}\right.}\right)+d \phi^{-1}\left(\frac{p(N+1)}{p\left(m_{2}\right.}\right)>0
$$

and

$$
\begin{aligned}
& G\left(\phi^{-1}\left(\frac{\sum_{j=0}^{N} f(j, x(j), \Delta x(j))}{\delta p(N+1)}\right)\right) \\
\geq & 1-c \phi^{-1}\left(\frac{(1+\delta) p(N+1)}{p\left(m_{1}\right)}\right)+d \phi^{-1}\left(\frac{p(N+1)}{p\left(m_{2}\right)}\right)=0 .
\end{aligned}
$$

Since $G(s)$ is increasing on $[0,+\infty)$, we get that $G(s)>0$ for all $s \in\left(\sigma_{f},+\infty\right)$. Then $F(s)>0$ for all $s \in\left(\sigma_{f},+\infty\right)$. Since (A1) implies that

$$
\begin{aligned}
& \lim _{s \rightarrow 0^{+}} F(s) s=-c \phi^{-1}\left(\frac{\sum_{j=m_{1}}^{N} f(j, x(j), \Delta x(j))}{p\left(m_{1}\right)}\right)+d \phi^{-1}\left(\frac{\sum_{j=m_{2}}^{N} h(j)}{p\left(m_{2}\right)}\right) \\
& \leq \frac{\phi^{-1}\left(\sum_{j=m_{2}}^{N} f(j, x(j), \Delta x(j))\right)}{\phi^{-1}(p(N+1))} \times \\
&\left(-c \phi^{-1}\left(\frac{p(N+1)}{p\left(m_{1}\right)}\right)+d \phi^{-1}\left(\frac{p(N+1)}{p\left(m_{2}\right)}\right)\right) \leq 0,
\end{aligned}
$$

we get that $\lim _{s \rightarrow 0^{+}} F(s) \leq 0$. Together with $F\left(\sigma_{f}\right) \geq G\left(\sigma_{f}\right) \geq 0$, we get that (11) holds. The proof is completed.

Define the operator $T: P \rightarrow X$ by

$$
(T x)(n)=B_{x}+\sum_{i=0}^{n-1} \phi^{-1}\left(\frac{p(N+1) \phi\left(A_{x}\right)+\sum_{j=i}^{N} f(j, x(j), \Delta x(j))}{p(i)}\right)
$$

for $n \in[0, N+2], x \in P$, where $A_{x}$ and $B_{x}$ are defined in Lemma 3.3.

Lemma 3.4. Suppose that (A1)-(A3) hold. Then 
(i) $T x \in P$ for each $x \in P$;

(ii) $x$ is a solution of $\operatorname{BVP}(6)$ if and only if $x$ is a solution of the operator equation $x=T x$;

(iii) $T: P \rightarrow P$ is completely continuous;

(iv) $T x$ satisfies

$$
\left\{\begin{array}{c}
\Delta[p(n) \phi(\Delta(T x)(n))]+f(n, x(n+1), \Delta x(n+1))=0, \quad n \in[0, N], \\
\alpha(T x)(0)-\beta \Delta(T x)(0)=-a(T x)\left(n_{1}\right)+b(T x)\left(n_{2}\right) \\
+a_{1} \Delta(T x)\left(n_{1}\right)-b_{1} \Delta(T x)\left(n_{2}\right), \\
\Delta(T x)(N+1)=c \Delta(T x)\left(m_{1}\right)-d \Delta(T x)\left(m_{2}\right) ;
\end{array}\right.
$$

(v) $A_{x}$ satisfies $A_{x} \in\left[0, \sigma_{f}\right]$.

Proof. The proofs of (i), (ii), (iv) and (v) are simple and omitted.

(iii). It suffices to prove that $T$ is continuous on $P$ and $T$ is relative compact. We divide the proof into three steps. These three steps imply that $T: P \rightarrow P$ is completely continuous. We omit the details.

Step 1. For each bounded subset $D \subset P$, prove that $\left\{\left(A_{x}, B_{x}\right): x \in \bar{D}\right\}$ is bounded in $R^{2}$.

Step 2. For each bounded subset $D \subset P$, and each $x_{0} \in D$, prove that $T$ is continuous at $x_{0}$. D.

Step 3. For each bounded subset $D \subset P$, prove that $T$ is pre-compact on

Proof of Theorem 2.1. We prove that all conditions in Lemma 3.1 are satisfied. It is easy to see that $\alpha_{1}, \alpha_{2}$ are two nonnegative continuous concave functionals on the cone $P, \beta_{1}, \beta_{2}, \beta_{3}$ are three nonnegative continuous convex functionals on the cone $P$. One sees from Lemma $3.2(\mathrm{v})$ that

$$
\max _{n \in[0, N+2]} x(n) \leq\left(N+2+\frac{\beta+a n_{1}+b n_{2}+a_{1}+b_{1}}{\alpha}\right) \max _{s \in[0, N+1]}|\Delta x(s)|
$$

for all $x \in P$. It follows that there exist constants $M>0$ such that

$$
\|y\|=\max \left\{\max _{n \in[0, N+2]}|y(n)|, \max _{n \in[0, N+1]}|\Delta y(n)|\right\} \leq M \beta_{1}(y) \text { for all } y \in P .
$$

Lemma 3.4 implies that $x=x(n)$ is a positive solution of $\mathrm{BVP}(6)$ if and only if $x(n)$ is a solution of the operator equation $x=T x$ and $T: P \rightarrow P$ is completely continuous. Then (1) and (2) in Lemma 3.1 hold. Now, we prove that (3) in Lemma 3.1 holds.

Choose

$$
a_{5}=c_{1}, \quad a_{4}=\frac{k}{N+2} e_{1}, \quad a_{3}=\frac{N+2}{k} e_{2}, \quad a_{2}=e_{2}, \quad a_{1}=e_{1} .
$$


Now, we prove that all conditions of Lemma 3.1 hold. One sees that $0<a_{1}<$ $a_{2}$. The remainder is divided into five steps.

Step 1. Prove that $T: \overline{P_{a_{5}}} \rightarrow \overline{P_{a_{5}}}$;

For $y \in \overline{P_{c_{1}}}$, we have $\|y\| \leq c_{1}$. Then $0 \leq y(t) \leq c_{1}$ for $t \in[0, N+$ 2] and $-c_{1} \leq \Delta y(t) \mid \leq c_{1}$ for all $n \in[0, N+1]$. So (A4) implies that $f(t, y(n), \Delta y(n)) \leq Q, t \in[0, N]$. Since

$$
Q=\phi\left(\frac{\alpha c_{1}}{\alpha N+2 \alpha+\beta+a n_{1}+b n_{2}+a_{1}+b_{1}}\right) \frac{\delta \min _{n \in[0, N]} p(n)}{(1+\delta)(N+1)},
$$

we have that

$$
\phi^{-1}\left(\frac{(1+\delta)(N+1) Q}{\delta \min _{n \in[0, N+1]} p(n)}\right) \leq c_{1}
$$

and

$$
\left(N+2+\frac{\beta+a n_{1}+b n_{2}+a_{1}+b_{1}}{\alpha}\right) \phi^{-1}\left(\frac{(1+\delta)(N+1) Q}{\delta \min _{n \in[0, N+1]} p(n)}\right) \leq c_{1} .
$$

Then

$$
\begin{aligned}
|\Delta(T y)(n)| & =\left|\phi^{-1}\left(\frac{p(N+1) \phi\left(A_{y}\right)+\sum_{i=n}^{N} f(i, y(i), \Delta y(n))}{p(n)}\right)\right| \\
& \leq \phi^{-1}\left(\frac{\frac{\sum_{i=0}^{N} f(i, y(i), \Delta y(n))}{\delta}+\sum_{i=n}^{N} f(i, y(i), \Delta y(n))}{p(n)}\right) \\
& \leq \phi^{-1}\left(\frac{(1+\delta) \sum_{n=0}^{N} f(n, y(n), \Delta y(n))}{\delta p(n)}\right) \\
& \leq \phi^{-1}\left(\frac{(1+\delta)(N+1) Q}{\delta \min _{n \in[0, N+1]} p(n)}\right) \leq c_{1} .
\end{aligned}
$$

On the other hand, we have from Lemma 3.4 that $T y \in P$. Lemma 3.3 implies $0 \leq A_{y} \leq \sigma_{f}$, then

$$
\begin{aligned}
0 & \leq(T y)(n) \leq\left(N+2+\frac{\beta+a n_{1}+b n_{2}+a_{1}+b_{1}}{\alpha}\right) \max _{s \in[0, N+1]}|\Delta(T y)(s)| \\
& \leq\left(N+2+\frac{\beta+a n_{1}+b n_{2}+a_{1}+b_{1}}{\alpha}\right) \phi^{-1}\left(\frac{(1+\delta)(N+1) Q}{\delta \min _{n \in[0, N+1]} p(n)}\right) \leq c_{1} .
\end{aligned}
$$

It follows that

$$
\|T y\|=\max \left\{\max _{t \in[0, N+2]}|(T y)(t)|, \max _{t \in[0, N+1]}|\Delta(T y)(t)|\right\} \leq c_{1} .
$$


Then $T: \overline{P_{c_{1}}} \rightarrow \overline{P_{c_{1}}}$. Hence (3)-(C1) holds.

Step 2. Prove that $\alpha_{1}(T y)>a_{2}$ for $y \in P\left(\beta_{1}, \alpha_{1} ; a_{2}, a_{5}\right)$ with $\beta_{3}(T y)>$ $a_{3}$;

For $y \in P\left(\beta_{1}, \alpha_{1} ; a_{2}, a_{5}\right)=P\left(\beta_{1}, \alpha_{1} ; e_{2}, c_{1}\right)$ with $\beta_{3}(T y)>b=\frac{e_{2}}{\sigma_{0}}$, we have that $\alpha_{1}(y) \geq e_{2}, \beta_{1}(y) \leq c_{1}$, and

$$
\max _{t \in[k, N+2]}(T y)(t)=(T y)(N+2)>\frac{(N+2) e_{2}}{k} .
$$

Hence Lemma 3.4(i) and Lemma 3.2(iv) imply that

$$
\alpha_{1}(T y)=\min _{t \in[k, N+2]}(T y)(t)=(T y)(k) \geq \frac{k}{N+2}(T y)(N+2)>e_{2}=a_{2} .
$$

This completes the proof of $(3)-(\mathrm{C} 2)$.

Step 3. Prove that $\beta_{2}(T y)<a_{1}$ for each $y \in Q\left(\beta_{1}, \beta_{2} ; a_{1}, a_{5}\right)$ with $\alpha_{2}(T y)<a_{4}$

For $y \in Q\left(\beta_{1}, \beta_{2} ; a_{1}, a_{5}\right)$ with $\alpha_{2}(T y)<a_{4}$, we have that $\beta_{1}(y) \leq a_{5}=c_{1}$, $\beta_{2}(y) \leq a_{1}=e_{1}$, and $\alpha_{2}(T y)<a_{4}=\frac{k}{N+2} e_{1}$. Then

$$
\beta_{2}(T y)=\max _{t \in[0, N+2]}(T y)(t)=(T y)(N+2) \leq \frac{N+2}{k} \min _{t \in[k, N+2]}(T y)(t)<e_{1}=a_{1} .
$$

This completes the proof of (3)-(C3).

Step 4. Prove that $\left\{y \in P\left(\beta_{1}, \beta_{3}, \alpha_{1} ; a_{2}, a_{3}, a_{5}\right): \alpha_{1}(x)>a_{2}\right\} \neq \emptyset$ and $\alpha_{1}(T y)>e_{2}$ for every $y \in P\left(\beta_{1}, \beta_{3}, \alpha_{1} ; e_{2}, \frac{N+2}{k} e_{2}, c_{1}\right)$;

It is easy to see that $\left\{y \in P\left(\beta_{1}, \beta_{3}, \alpha_{1} ; e_{2}, \frac{N+2}{k} e_{2}, c_{1}\right): \alpha_{1}(y)>e_{2}\right\} \neq \emptyset$.

For $y \in P\left(\beta_{1}, \beta_{3}, \alpha_{1} ; e_{2}, \frac{N+2}{k} e_{2}, c_{1}\right)$, one has that $\alpha_{1}(y) \geq e_{2}, \beta_{3}(y) \leq$ $(N+2) e_{2} / k, \beta_{1}(y) \leq c_{1}$. Then

$$
e_{2} \leq y(t) \leq \frac{N+2}{k} e_{2}, t \in[k, N+2],|\Delta y(t)| \leq c_{1} .
$$

Thus (A5) implies that $f(t, y(t), \Delta y(t)) \geq W, t \in[k, N]$. Since

$$
\alpha_{1}(T y)=\min _{t \in[k, N+2]}(T y)(t) \geq \frac{k}{N+2} \max _{t \in[0, N+2]}(T y)(t),
$$

we get

$$
\alpha_{1}(T y) \geq \frac{k}{N+2}(T y)(N+2)
$$


It follows from Lemma 3.3 that

$$
\begin{aligned}
\alpha_{1}(T y) & \geq \frac{k}{N+2}(T y)(N+2) \\
& \left.=\frac{k\left[B_{y}+\sum_{i=0}^{N+1} \phi^{-1}\left(\frac{p(N+1) \phi\left(A_{y}\right)+\sum_{j=i}^{N} f(j, y(j), \Delta y(j))}{p(i)}\right)\right]}{N+2}\right) \\
& \geq \frac{k}{N+2} \sum_{i=0}^{k} \phi^{-1}\left(\frac{\sum_{j=k}^{N} f(j, y(j), \Delta y(j))}{p(i)}\right) \\
& >\frac{k}{N+2} \sum_{i=0}^{k} \phi^{-1}\left(\frac{(N-k+1) W}{p(i)}\right)=e_{2} .
\end{aligned}
$$

This completes the proof of (3)-(C4).

Step 5. Prove that $\left\{y \in Q\left(\beta_{1}, \beta_{3}, \alpha_{2} ; a_{4}, a_{1}, a_{5}\right): \beta_{2}(x)<a_{1}\right\} \neq \emptyset$ and $\beta_{2}(T x)<a_{1}$ for every $x \in Q\left(\beta_{1}, \beta_{3}, \alpha_{2} ; a_{4}, a_{1}, a_{5}\right)$;

It is easy to see that $\left\{y \in Q\left(\beta_{1}, \beta_{3}, \alpha_{2} ; a_{4}, a_{1}, a_{5}\right): \beta_{2}(x)<a_{1}\right\} \neq \emptyset$. For $y \in Q\left(\beta_{1}, \beta_{3}, \alpha_{2} ; a_{4}, a_{1}, a_{5}\right)$, one has that $\alpha_{2}(y)=\geq a_{4}=k e_{1} /(N+2)$, $\beta_{3}(y)=\leq a_{1}=e_{1}, \beta_{1}(y) \leq a_{5}=c_{1}$. Hence we get that $0 \leq y(t) \leq e_{1}, t \in$ $[0, N+2] ; \quad-c \leq \Delta y(t) \leq c_{1}, t \in[0, N+1]$. Then (A 6$)$ implies that $f(t, y(t), \Delta y(t)) \leq E, \quad t \in[0, N]$. Similarly to Step 1 , we get that

$$
\begin{aligned}
\beta_{2}(T y) & =\max _{n \in[0, N+2]}(T y)(n)=(T y)(N+2) \\
& \leq\left(N+2+\frac{\beta+a n_{1}+b n_{2}+a_{1}+b_{1}}{\alpha}\right) \max _{s \in[0, N+1]}|\Delta(T y)(s)| \\
& \leq\left(N+2+\frac{\beta+a n_{1}+b n_{2}+a_{1}+b_{1}}{\alpha}\right) \phi^{-1}\left(\frac{(1+\delta)(N+1) E}{\delta \min _{n \in[0, N+1]} p(n)}\right) \\
& \leq e_{1}=a_{1} .
\end{aligned}
$$

This completes the proof of (3)-(C5). Then Lemma 3.1 implies that $T$ has at least three fixed points $y_{1}, y_{2}$ and $y_{3}$ such that $\beta_{2}\left(y_{1}\right)<e_{1}, \alpha_{1}\left(y_{2}\right)>$ $e_{2}, \beta_{2}\left(y_{3}\right)>e_{1}, \alpha_{1}\left(y_{3}\right)<e_{2}$. Hence $\operatorname{BVP}(6)$ has three increasing positive solutions $x_{1}, x_{2}$ and $x_{3}$ such that $x_{1}(N+2)<e_{1}, x_{2}(k)>e_{2}, x_{3}(N+2)>$ $e_{1}, x_{3}(k)<e_{2}$. The proof is complete.

Acknowledgments. The author would like to thank the anonymous referees for their careful reading of this manuscript and for suggesting useful rhetorical changes. 


\section{References}

[1] D.R. Anderson, Discrete third-order three-point right-focal boundary value problems, Comput. Math. Appl. 45(2003) 861-871.

[2] D. Anderson, R.I. Avery, Multiple positive solutions to a third-order discrete focal boundary value problem, Comput. Math. Appl. 42(2001) 333340 .

[3] R.I. Avery, A.C. Peterson, Three positive fixed points of nonlinear operators on ordered Banach spaces, Comput. Math. Appl. 42(2001) 313-322.

[4] N. Aykut, Existence of positive solutions for boundary value problems of second-order functional difference equations, Comput. Math. Appl. 48(2004) 517-527.

[5] P. Candito, N. Giovannelli, Multiple solutions for a discrete boundary value problem involving the p-Laplacian, Comput. Math. Appl., 56(2008) 959-964.

[6] W. Cheung, J. Ren, P.J.Y. Wong, D. Zhao, Multiple positive solutions for discrete nonlocal boundary value problems, J. Math. Anal. Appl. 330(2007) 900-915.

[7] X. Cai, J. Yu, Existence theorems for second-order discrete boundary value problems, J. Math. Anal. Appl. 320(2006) 649-661.

[8] J. R. Graef and J. Henderson, Double solutions of boundary value problems for $2 \mathrm{mth}$-order differential equations and difference equations, Comput. Math. Appl. 45(2003) 873-885.

[9] Z. He, On the existence of positive solutions of p-Laplacian difference equations, J. Comput. Appl. Math. 161(2003) 193-201.

[10] I.Y. Karaca, Discrete third-order three-point boundary value problem, J. Comput. Appl. Math. 205(2007) 458-468.

[11] R. Leggett, L. Williams, Multiple positive fixed points of nonlinear operators on ordered Banach spaces, Indiana Univ. Math. J., 28(1979) 673-688.

[12] Y. Li, L. Lu, Existence of positive solutions of p-Laplacian difference equations, Appl. Math. Letters 19(2006)1019-1023.

[13] Y. Liu, W. Ge, Twin positive solutions of boundary value problems for finite difference equations with p-Laplacian operator, J. Math. Anal. Appl. 278(2003) 551-561. 
[14] Y. Liu, Positive solutions of multi-point BVPs for second order pLaplacian difference equations. Commun. Math. Anal. 4 (2008) 58-77.

[15] R. Ma, Multiple positive solutions for nonlinear m-point boundary value problems. Appl. Math. Comput. 148(2004) 249-262.

[16] R. Ma, B. Thompson, Positive solutions for nonlinear m-point eigenvalue problems. J. Math. Anal. Appl. 297(2004) 24-37.

[17] H. Pang, H. Feng, W. Ge, Multiple positive solutions of quasi-linear boundary value problems for finite difference equations, Appl. Math. Comput. 197(2008) 451-456.

[18] D. Wang, W. Guan, Three positive solutions of boundary value problems for p-Laplacian difference equations, Comput. Math. Appl., 55(2008) 1943-1949.

[19] P.J.Y. Wong, R.P. Agarwal, Existence theorems for a system of difference equations with (n,p)-type conditions, Appl. Math. Comput. 123(2001) 389-407.

[20] P.J.Y. Wong, L. Xie, Three symmetric solutions of lidstone boundary value problems for difference and partial difference equations, Comput. Math. Appl. 45(2003) 1445-1460.

[21] F. Xu, Positive solutions for multipoint boundary value problems with one-dimensional p-Laplacian operator, Appl. Math. Comput. 194(2007) 366-380.

[22] C. Yang, P. Weng, Green functions and positive solutions for boundary value problems of third-order difference equations, Comput. Math. Appl. $54(2007) 567-578$.

[23] G. Zhang, R. Medina, Three-point boundary value problems for difference equations, Comput. Math. Appl. 48(2004) 1791-1799.

Yuji Liu,

Department of Mathematics,

Guangdong University of Business Studies,

Guangzhou, 510320, P R China.

Email: liuyuji888@sohu.com 
